\title{
Differential transforming activity of the retroviral Tax oncoproteins in humanT lymphocytes
}

\author{
Tong Ren ${ }^{1}$ and Hua Cheng ${ }^{2}$ * \\ 1 Penn State Hershey Cancer Institute, Hershey, PA, USA \\ ${ }^{2}$ Institute of Human Virology, School of Medicine, University of Maryland, Baltimore, MD, USA
}

\author{
Edited by: \\ Umberto Bertazzoni, University of \\ Verona, Italy \\ Reviewed by: \\ Masanori Baba, Kagoshima \\ University, Japan \\ Edward Harhaj, Johns Hopkins School \\ of Medicine, USA \\ Masahiro Fujii, Niigata University \\ Graduate School of Medical and \\ Dental sciences, Japan \\ *Correspondence: \\ Hua Cheng, Institute of Human \\ Virology, School of Medicine, \\ University of Maryland, 655 West \\ Baltimore Street, Baltimore, MD \\ 21201, USA \\ e-mail: hcheng@ihv.umaryland.edu
}

\begin{abstract}
Human T cell leukemia virus type 1 and type 2 (HTLV-1 and -2) are two closely related retroviruses. HTLV-1 causes adult T cell leukemia and lymphoma, whereas HTLV-2 infection is not etiologically linked to human disease. The viral genomes of HTLV-1 and -2 encode highly homologous transforming proteins, Tax-1 and Tax-2, respectively. Tax-1 is thought to play a central role in transforming CD4+ T lymphocytes. Expression of Tax-1 is crucial for promoting survival and proliferation of virally infected human T lymphocytes and is necessary for initiating HTLV-1-mediated oncogenesis. In transgenic mice and humanized mouse model, Tax-1 has proven to be leukemogenic. Although Tax-1 is able to efficiently transform rodent fibroblasts and to induce lymphoma in mouse model, it rarely transforms primary human CD4+ T lymphocytes. In contrast, Tax-2 efficiently immortalizes human $\mathrm{CD} 4+\mathrm{T}$ cells though it exhibits a lower transforming activity in rodent cells as compared to Tax-1. We here discuss our recent observation and views on the differential transforming activity of Tax-1 and Tax-2 in human T cells.
\end{abstract}

Keywords: HTLV-1/-2, Tax, human T lymphocytes, immortalization, transformation
HTLV-1 and -2 are two highly homologous human retroviruses. Both viruses are blood-borne pathogens, which can be transmitted through perinatal transmission by blood contamination or breast milk feeding, sexual transmission, exposure to contaminated blood products or sharing contaminated needles (Clark et al., 1985; The T- and B-cell Malignancy Study Group, 1988). Although both HTLV-1 and -2 utilize GLUT-1, a ubiquitously expressed cell surface (SU) protein, as the receptor for viral entry (Igakura et al., 2003; Manel et al., 2003; Coskun and Sutton, 2005), they exhibit distinct cell tropism. HTLV-1 preferentially infects and transforms CD4+ T cells, whereas HTLV-2 has tropism for CD8+ $\mathrm{T}$ cells, which suggests that there exists a CD8+ T cell-specific co-factor for assisting viral entry and permissive replication of HTLV-2 (Ijichi et al., 1992). HTLV-1 is the etiological factor that causes adult T cell leukemia and lymphoma (Hattori et al., 1981; Hinuma et al., 1981, 1982; Yamaguchi et al., 1984). HTLV-2 was detected in CD8 T cells from a patient with hairy cell leukemia, a rare type of leukemia that affects B lymphocytes (Kalyanaraman et al., 1982), however the causative link between HTLV-2 infection and hairy cell leukemia has not been established. Thus far, HTLV-2 infection has not been etiologically linked to any human disease.

HTLV-1 viral genome is known to encode two oncogenic products, Tax-1 and HBZ (Satou et al., 2006; Satou and Matsuoka, 2012). Tax-1 is the first recognized viral component to display oncogenic potential. Evidence showed that Tax-1 plays an essential role in mediating transformation of $\mathrm{T}$ lymphocytes (Tanaka et al., 1990). The transforming activity of Tax-1 in lymphocytes was first demonstrated using HTLV-1 infectious molecular clone. The wild type molecular clone efficiently transformed human primary $\mathrm{T}$ cells, while the molecular clone with disrupted tax gene failed to do so, defining the role of Tax-1 as a viral transforming protein (Yamaoka et al., 1996, 1998; Akagi et al., 1997; Matsumoto et al., 1997; Iwanaga et al., 1999). However, this experiment did not rule out potential participation of other viral components in HTLV-1-mediated cell transformation. Unexpectedly, when expressed alone, Tax-1 rarely immortalizes or transforms terminally differentiated $\mathrm{T}$ cells derived from human peripheral blood (Bellon et al., 2010). In humanized mouse model, Tax-1-expressing human CD34+ blood progenitor cells, when transplanted into NOD/SCID mice, developed T cell lymphoma of human origin (Banerjee et al., 2010). This experimental finding suggests that in some cases, leukemic cells might be evolved from HTLV-1-infected CD34+ blood progenitor cells rather than from infected mature T cells. This assumption is yet to be validated.

A comparative study on two highly homologous Tax proteins from HTLV-1 and - 2 is crucial in deciphering the molecular pathogenesis of HTLV-1-associated ATL. We expressed the Tax proteins in human $\mathrm{T}$ cells isolated from peripheral blood to imitate the process of HTLV-1 infection. This prospective approach would allow us to evaluate sequential oncogenic events in Tax-mediated proliferation, immortalization and transformation of human primary T cells. Normal lymphocytes isolated from healthy donors are typically at quiescent stage. Stimulation with mitogens such as PHA, recombinant IL-2 or mitogenic anti-CD3/-CD28 antibodies results in rapid proliferation of peripheral blood lymphocytes with displaying lymphoblastic morphology. Activated lymphocytes stop growing 2-3 weeks following stimulation at normal culture conditions by reaching the checkpoint of $\mathrm{T}$ cell senescence. HTLV-1 infection overrides replicative senescence of T cells, promotes continuous growth of HTLV-1-infected T cells and induces immortalization, ultimately resulting in IL-2-independent growth of the transformed $\mathrm{T}$ cells. At each step of aberrant proliferation, 
immortalization or transformation during HTLV-1 infection of $\mathrm{T}$ cells, a variety of cellular oncogenic alterations are expected to occur. Thus, studying these processes will provide crucial insights into the pathogenesis of ATL.

We previously hypothesized that in the absence of other viral proteins, Tax-1 is sufficient to immortalize human mature CD4+ T cells while Tax- 2 preferentially immortalizes CD8+ T cells. We unexpectedly found that both Tax- 1 and Tax- 2 failed to immortalize human primary CD8 $+\mathrm{T}$ cells, and Tax- 2 was able to immortalize human CD4 $+\mathrm{T}$ cells more efficiently than Tax-1 in the study with including 12 healthy blood donors. A similar finding was reported later (Imai et al., 2013). In addition, a majority of Tax-2-immortalized CD4+ $\mathrm{T}$ cell lines grew in vitro at the growth rate similar to some lymphoblastic leukemia cells (Ren et al., 2012). In contrast, Tax-1-immortalized CD4+ T cells were slow growing and exhibited spontaneous cell death at normal culture conditions (Ren et al., unpublished data). These experimental results were unlikely caused by technical variation because Tax-1 and Tax-2, in which their expressions were driven from human elongation factor promoter, were introduced into human primary T cells via VSV-G pseudotyped lentiviruses at similar efficiency.

Tax-2 appears to be more oncogenic than Tax-1 in primary CD4+ $\mathrm{T}$ cells when they are expressed alone, however Tax-1 is clearly oncogenic in CD4 $+\mathrm{T}$ cells in the context of an intact proviral clone and other expressed viral proteins. Although both Tax proteins are highly homologous, Tax-1 does exhibit distinct structural features in which Tax-2 lacks. Tax-1 contains a PDZ binding motif (PBM) in its carboxyl-terminus that is important for binding to DLG-1 and other PDZ containing proteins, and these interactions were thought to play an important role for cell transformation by Tax-1. In addition, Tax-1, but not Tax-2, undergoes K63-linked polyubiquitination as part of its mechanism to activate NF-кB (Shembade et al., 2007; Journo et al., 2013). It is apparent that these differences do not account for the stronger transforming capability of Tax- 2 in primary CD $4+\mathrm{T}$ cells. Tax- 1 may acquire a full transforming ability in human $\mathrm{CD} 4+\mathrm{T}$ cells in cooperation with HBZ, an HTLV-1 antisense gene product that is constitutively transcribed and remains intact in ATL cells. In fact, HBZ itself was proven leukemogenic in mouse model. 30\% of HBZ-transgenic (Tg) mice developed T cell lymphoma and most of HBZ Tg mice developed spontaneous inflammatory lesions (Satou and Matsuoka, 2012). Furthermore, increasing evidences showed that the functional interaction between Tax and HBZ is crucial for HTLV-1 oncogenesis in patients. Since high levels of Tax-1 expression lead to hyper-activation of NF- $\kappa \mathrm{B}$, consequently resulting in replicative senescence or even cell death (Zhi et al., 2011), HBZ antagonizes Tax-1's toxic function to facilitate immune evasion and to promote cell cycle progression and proliferation. Interestingly, Tax-2 also induces hyper-activation of NF- $\kappa \mathrm{B}$ in $\mathrm{CD} 4+\mathrm{T}$ cells, and it is capable of promoting $\mathrm{T}$ cell proliferation in the absence of the antisense gene product encoded from HTLV-2. This suggests that unlike Tax-1, Tax-2 is a singly important viral component of the HTLV-2 genome to execute its transforming activity in CD4+ T cells.

We also found that the Tax proteins had their own preference on donor selection, and Tax- 1 and Tax- 2 immortalized $\mathrm{CD} 4+\mathrm{T}$ cells from different blood donors. In one unusual case, Tax-1 immortalized CD4+ $\mathrm{T}$ cells while Tax-2 immortalized CD4+ myeloid dendritic cells (mDCs) from CD4+ cell pools of the same blood donor (Ren et al., unpublished data). These mDCs showed negative lineage markers (CD3-/TCR $\alpha \beta$ /TCR $\gamma \delta-/ C D 19-/ C D 56-/ C D 16-/ C D 14-/ C D 34-)$ and positive myeloid/dendritic cell markers (CD11c+/CD123+/HLA$\mathrm{DR}+/ \mathrm{CD} 2+/ \mathrm{CD} 71+/ \mathrm{CD} 117+/ \mathrm{CD} 33+)$. Because the number of $\mathrm{mDCs}$ in healthy donors is typically less than $1 \%$, the finding that Tax-2 is able to immortalize mDCs from enriched CD4+ pools suggests that the Tax-2-mediated immortalization of blood cells is highly selective. Aside from this finding, genotyping analysis of one Tax-2-established CD4 T cell line showed that these cells were clonal population. Together, our data demonstrate that in the absence of other viral components, Tax-2 alone is sufficient to promote clonal expansion of selected subsets of human primary CD4+ T cells. The potential cellular factor that contributes to Tax's selectivity is currently unclear, which will certainly be an interesting subject for further investigation.

It is known that HTLV-1 has preferential tropism for CD4 T cells in healthy carriers, HAM/TSP and ATL patients. In addition to utilization of GLUT1 as an entry factor for HTLV viruses, both viruses also utilize NRP1. HTLV-1 and -2 have a differential requirement for heparan sulfate proteoglycans (HSPGs). Distinct from HTLV1, HTLV-2 is not dependent on HSPGs for cell entry (Jones et al., 2006). Because HTLV-1 utilizes a ubiquitously expressed receptor for viral entry, it is expected that this virus would have the capacity to infect cell types other than CD4+ T cells. Indeed, CD8+ lymphocytes, monocytes and B-lymphocytes are found to harbor HTLV-1 (Koyanagi et al., 1993; Eiraku et al., 1998; Nagai et al., 2001). In addition, macrophages, dendritic cells, megakaryocytes as well as glial cells (astrocytes and microglial cells) are also the cell targets for HTLV-1 infection in vivo (Macatonia et al., 1992; Koyanagi et al., 1993; Levin et al., 1997; Grant et al., 2002). HTLV-1 isolates can be transmitted to primary human endothelial cells and basal mammary epithelial cells in vitro (Ho et al., 1984; Hoxie et al., 1984). Regardless of a broad cell tropism of HTLV-1, this virus exclusively causes malignant transformation of infected CD4+ T cells, suggesting that a panel of CD4 cell-specific cellular factors is crucial in assisting HTLV-1-mediated oncogenesis. Conversely, it is also possible that other types of cells may express cellular repressors to restrict HTLV-1 oncogenesis as seen in other retroviruses. For instance, SAMHD1 is able to restrict HTLV-1 replication in myeloid cells (St. Gelais and Wu, 2011). Unlike HTLV-1, HTLV-2 preferentially infects CD8+ T cells in human. However, Tax-2, when expressed by a pseudotype form of lentivirus, executes its transforming activity in CD4+ T cells. This phenomenon of disintegrated cell tropism (in CD8 $+\mathrm{T}$ cells) and disease-causing capacity (in CD4+ T cells) may partially explain why HTLV-2 does not currently cause leukemia in human.

These findings may have important implications on pathogenic virus evolution. Many viruses are considered non-pathogenic, because they do not apparently cause human disease based on current data. These non-pathogenic viruses usually do not receive much attention as known pathogenic viruses do. From an evolutionary point of view, recombination and cell tropism switch among homologous pathogenic and non-pathogenic viruses may occur, potentially generating a more lethal virus. A recent study 
demonstrated that cell tropism switch could be initiated by artificially generating chimeric envelope proteins. Switch of the envelope SU domains between HTLV-1 and HTLV-2 alters the tropism of HTLV-1 from CD4 to CD8+ T cells (Kannian et al., 2013). In the case of HTLV-2, the infection rate among IV drug abusers is as high as $30 \%$. Since no treatment is available for HTLV2 infection and host immunity is not sufficient to eradicate this virus, it is possible that the rate of infection is going to rise due to the presence of accumulative HTLV-2 reservoirs in host CD8+ T cells. Indeed, co-infection of HTLV-2 with HTLV-1 or with HIV1 has been documented and is increasing (Uchiyama et al., 1977; Shimoyama, 1991), probably because these viruses share similar transmission pathways and risk factors. HTLV-2 could be eventually modified in dendritic cells or monocytes that are co-infected with HTLV-1 or HIV-1 to acquire new cell tropism for CD4+ T cells, leading to its transformation of CD4+ T cells. Although naturally occurring cell tropism switch among homologous viruses may take unpredictable amount of time, this event does occur evolutionarily.

Although Tax-2 does not transform normal CD8 T cells, this viral protein exhibits its transforming activity in CD8 $+\mathrm{T}$ cells derived from $\mathrm{T}$ cell type of large granular lymphocyte leukemia (T-LGLL). T-LGL leukemia is the malignancy of CD8+ cytotoxic T cells, which usually occurs in elderly patients. The disease course is frequently associated with autoimmune diseases (Rose and Berliner, 2004). Primary T-LGL leukemia cells display the $\mathrm{CD} 3+/ \mathrm{CD} 8+/ \mathrm{CD} 57+$ SU markers, representing activated cytotoxic T lymphocytes. Despite T-LGLL cells are leukemic cells, these cells do not grow in culture. By expressing Tax-2 in these cells, long-term growth and clonal expansion of T-LGLL cells could be achieved (Ren et al., unpublished data).

In summary (see Table 1), Tax-1 and Tax-2 display differential transforming activities in human T lymphocytes. Tax-2 demonstrates a more potent activity than Tax- 1 in immortalizing human terminally differentiated CD4+ T cells. Besides, Tax-2, not Tax1 , is able to transform CD8 $+\mathrm{T}$ cells from T-LGL leukemic cells with preexisting oncogenic profile. The ability of Tax- 2 to establish specific subsets of $\mathrm{T}$ cell lines implicates its utilization in studying

\section{REFERENCES}

Akagi, T., Ono, H., Nyunoya, H., and Shimotohno, K. (1997). Characterization of peripheral blood T-lymphocytes transduced with HTLV-I Tax mutants with different trans-activating phenotypes. Oncogene 14, 2071-2078. doi: 10.1038/sj.onc. 1201045

Banerjee, P., Tripp, A., Lairmore, M. D., Crawford, L., Sieburg, M., Ramos, J. C., et al. (2010). Adult T-cell leukemia/lymphoma development in HTLV-1-infected humanized SCID mice. Blood 115, 2640-2648. doi: 10.1182/blood-2009-10-246959

Bellon, M., Baydoun, H. H., Yao, Y., and Nicot, C. (2010). HTLV-I Taxdependent and -independent events associated with immortalization of human primary $\mathrm{T}$ lymphocytes. laer, K., etal. (1985). Seroepi-

Table 1 | Differential activities of Tax-1 and Tax-2 in human T cells.

\begin{tabular}{lcc}
\hline & Tax-1 & Tax-2 \\
\hline Activation of & + & + \\
IKK/NF-kB & + & + \\
Stat3 & + & + \\
PI3K/Akt & + & + \\
AP-1 & + & + \\
CREB & + & + \\
Dysregulation of autophagy & $+^{a}$ & ++ \\
Lipid raft involvement & & - \\
Immortalization of & $+^{\mathrm{b}}$ & + \\
CD4+ T cells & - & + \\
CD8+ T cells & - & + \\
CD8+ T cells from T-LGLL &
\end{tabular}

a Tax-1 recruits $/ \kappa B$ kinases into lipid raft microdomains for persistent activation of $N F-\kappa B$ signaling, while Tax-2 activation of $N F_{-\kappa} B$ does not appear to involve lipid rafts (Huang etal., 2009).

${ }^{\mathrm{b}}$ Tax-1 is able to immortalize human primary CD4+ T cells with low efficiency 12 out of 12), and Tax-1-immortalized T cells grow slowly in culture and experience spontaneous cell death.

'Tax-2 immortalizes human primary CD4+ T cells more efficiently than Tax-1 (4 out of 12). Tax-2-immortalized T cells grow in culture at a rate comparable to some lymphoblastic leukemia cells with healthy growth appearance.

human T cell biology and in developing leukemia model. Furthermore, the hypothesis that Tax- 2 has a disease-causing capacity in $\mathrm{CD} 4+\mathrm{T}$ cells needs to be validated in humanized mouse model.

\section{ACKNOWLEDGMENTS}

Research reported in this publication was supported by the National Institute of Allergy And Infectious Diseases of the National Institutes of Health under award number R01AI090113 to Hua Cheng. The content is solely the responsibility of the authors and does not necessarily represent the official views of the National Institutes of Health.

individuals. J. Immunol. 161, 66746680. 10.1182/blood-2009-08-241117

Clark, J., Saxinger, C., Gibbs, W. N., Lofters, W., Lagranade, L., Deceudemiologic studies of human T-cell leukemia/lymphoma virus type I in Jamaica. Int. J. Cancer 36, 37-41. doi: 10.1002/ijc.2910360107

Coskun, A. K., and Sutton, R. E. (2005). Expression of glucose transporter 1 confers susceptibility to human T-cell leukemia virus envelope-mediated fusion. J. Virol. 79, 4150-4158. doi: 10.1128/JVI.79.7.4150-4158.2005

Eiraku, N., Hingorani, R., Ijichi, S., Machigashira, K., Gregersen, P. K., Monteiro, J., et al. (1998). Clonal expansion within CD4+ and $\mathrm{CD} 8+\mathrm{T}$ cell subsets in human $\mathrm{T}$ lymphotropic virus type I-infected
Grant, C., Barmak, K., Alefantis, T., Yao, J., Jacobson, S., and Wigdahl, B. (2002). Human T cell leukemia virus type I and neurologic disease: events in bone marrow, peripheral blood, and central nervous system during normal immune surveillance and neuroinflammation. J. Cell. Physiol. 190, 133-159. doi: 10.1002/jcp.10053

Hattori, T., Uchiyama, T., Toibana, T., Takatsuki, K., and Uchino, H. (1981). Surface phenotype of Japanese adult T-cell leukemia cells characterized by monoclonal antibodies. Blood 58 645-647.

Hinuma, Y., Komoda, H., Chosa, T., Kondo, T., Kohakura, M., Takenaka, T., et al. (1982). Antibodies to adult T-cell leukemia-virus-associated antigen (ATLA) in sera from patients with ATL and controls in Japan: a nation-wide sero-epidemiologic study. Int. J. Cancer 29, 631-635. doi: 10.1002/ijc. 2910290606

Hinuma, Y., Nagata, K., Hanaoka, M., Nakai, M., Matsumoto, T., Kinoshita, K. I., et al. (1981). Adult T-cell leukemia: antigen in an ATL cell line and detection of antibodies to the antigen in human sera. Proc. Natl. Acad. Sci. U.S.A. 78, 6476-6480. doi: 10.1073/pnas.78.10.6476

Ho, D. D., Rota, T. R., and Hirsch, M. S. (1984). Infection of human endothelial cells by human T-lymphotropic virus type I. Proc. Natl. Acad. Sci. U.S.A. 81, 7588-7590. doi: 10.1073/pnas.81.23.7588

Hoxie, J. A., Matthews, D. M., and Cines, D. B. (1984). Infection of 
human endothelial cells by human Tcell leukemia virus type I. Proc. Natl. Acad. Sci. U.S.A. 81, 7591-7595. doi: 10.1073/pnas.81.23.7591

Huang, J., Ren, T., Guan, H., Jiang, Y., and Cheng, H. (2009). HTLV1 Tax is a critical lipid raft modulator that hijacks IkappaB kinases to the microdomains for persistent activation of NF-kappaB. J. Biol. Chem. 284, 6208-6217. doi: 10.1074/jbc.M806390200

Igakura, T., Stinchcombe, J. C., Goon, P. K., Taylor, G. P., Weber, J. N., Griffiths, G. M., etal. (2003). Spread of HTLV-I between lymphocytes by virus-induced polarization of the cytoskeleton. Science 299, 17131716. doi: 10.1126/science.1080115

Ijichi, S., Ramundo, M. B., Takahashi, H., and Hall, W. W. (1992). In vivo cellular tropism of human $\mathrm{T}$ cell leukemia virus type II (HTLVII). J. Exp. Med. 176, 293-296. doi: 10.1084/jem.176.1.293

Imai, M., Higuchi, M., Kawamura, H., Yoshita, M., Takahashi, M., Oie, M., etal. (2013). Human T cell leukemia virus type 2 (HTLV-2) Tax2 has a dominant activity over HTLV1 Tax1 to immortalize human CD4+ T cells. Virus Genes 46, 39-46. doi: 10.1007/s11262-012-0831-9

Iwanaga, Y., Tsukahara, T., Ohashi, T., Tanaka, Y., Arai, M., Nakamura, M., et al. (1999). Human T-cell leukemia virus type 1 tax protein abrogates interleukin-2 dependence in a mouse T-cell line. J. Virol. 73, 1271-1277.

Jones, K. S., Fugo, K., Petrow-Sadowski, C., Huang, Y., Bertolette, D. C., Lisinski, I., et al. (2006). Human Tcell leukemia virus type 1 (HTLV-1) and HTLV-2 use different receptor complexes to enter T cells. J. Virol. 80, 8291-8302. doi: 10.1128/JVI. 00389-06

Journo, C., Bonnet, A., Favre-Bonvin, A., Turpin, J., Vinera, J., Côté, E., et al. (2013). Human T cell leukemia virus type 2 tax-mediated NF- $\kappa$ B activation involves a mechanism independent of Tax conjugation to ubiquitin and SUMO. J. Virol. 87, 1123-1136. doi: 10.1128/JVI.01792-12

Kalyanaraman, V. S., Sarngadharan, M. G., Robert-Guroff, M., Miyoshi, I., Golde, D., and Gallo, R. C. (1982). A new subtype of human T-cell leukemia virus (HTLV-II) associated with a T-cell variant of hairy cell leukemia. Science 218, 571-573. doi: 10.1126/science.6981847

Kannian, P., Fernandez, S., Jones, K. S., and Green, P. L. (2013). Human $\mathrm{T}$ lymphotropic virus type $1 \mathrm{SU}$ residue 195 plays a role in determining the preferential CD4+ $\mathrm{T}$ cell immortalization/transformation tropism. J. Virol. 87, 9344-9352. doi: 10.1128/JVI.01079-13

Koyanagi, Y., Itoyama, Y., Nakamura, N., Takamatsu, K., Kira, J., Iwamasa T., et al. (1993). In vivo infection of human T-cell leukemia virus type I in non-T cells. Virology 196, 25-33. doi: 10.1006/viro.1993.1451

Levin, M. C., Krichavsky, M., Fox, R. J., Lehky, T., Jacobson, S., Fox, C., et al. (1997). Extensive latent retroviral infection in bone marrow of patients with HTLV-I-associated neurologic disease. Blood 89, 346-348.

Macatonia, S. E., Cruickshank, J. K., Rudge, P., and Knight, S. C. (1992). Dendritic cells from patients with tropical spastic paraparesis are infected with HTLV-1 and stimulate autologous lymphocyte proliferation. AIDS Res. Hum. Retroviruses 8, 1699 1706. doi: 10.1089/aid.1992.8.1699

Manel, N., Kim, F. J., Kinet, S. Taylor, N., Sitbon, M., and Battini, J. L. (2003). The ubiquitous glucose transporter GLUT-1 is a receptor for HTLV. Cell 115, 449459. doi: 10.1016/S0092-8674(03) 00881-X

Matsumoto, K., Shibata, H., Fujisawa, J. I., Inoue, H., Hakura, A., Tsukahara, T., et al. (1997). Human T-cell leukemia virus type 1 Tax protein transforms rat fibroblasts via two distinct pathways. J. Virol. 71, 44454451.

Nagai, M., Brennan, M. B., Sakai, J. A., Mora, C. A., and Jacobson, S. (2001). $\mathrm{CD} 8(+) \mathrm{T}$ cells are an in vivo reservoir for human T-cell lymphotropic virus type I. Blood 98, 1858-1861. doi: 10.1182/blood.V98.6.1858

Ren, T., Dong, W., Takahashi, Y., Xiang, D., Yuan, Y., Liu, X., et al. (2012). HTLV-2 Tax immortalizes human CD4+ memory $\mathrm{T}$ lymphocytes by oncogenic activation and dysregulation of autophagy. J. Biol. Chem. 287, 34683-34693. doi: 10.1074/jbc.M112.377143

Rose, M. G., and Berliner, N. (2004). T-cell large granular lymphocyte leukemia and related disorders.
Oncologist 9, 247-258. doi: 10.1634/theoncologist.9-3-247

Satou, Y., and Matsuoka, M. (2012). Molecular and cellular mechanism of leukemogenesis of ATL: emergent evidence of a significant role for HBZ in HTLV-1-induced pathogenesis. Leuk. Res. Treatment 2012, 213653. doi: 10.1155/2012/ 213653

Satou, Y., Yasunaga, J., Yoshida, M., and Matsuoka, M. (2006). HTLV-I basic leucine zipper factor gene mRNA supports proliferation of adult $\mathrm{T}$ cell leukemia cells. Proc. Natl. Acad. Sci. U.S.A. 103, 720-725. doi: 10.1073/pnas.0507631103

Shembade, N., Harhaj, N. S., Yamamoto, M., Akira, S., and Harhaj, E. W. (2007). The human T-cell leukemia virus type 1 Tax oncoprotein requires the ubiquitinconjugating enzyme Ubc13 for NF-kappaB activation. J. Virol. 81, 13735-13742. doi: 10.1128/JVI.017 90-07

Shimoyama, M. (1991). Diagnostic criteria and classification of clinical subtypes of adult T-cell leukaemialymphoma. A report from the Lymphoma Study Group (1984-87). Br. J. Haematol. 79, 428-437. doi: 10.1111/j.1365-2141.1991.tb08051.x

St. Gelais, C., and Wu, L. (2011) SAMHD1: a new insight into HIV-1 restriction in myeloid cells. Retrovirology 8, 55. doi: 10.1186/1742-46908-55

Tanaka, A., Takahashi, C., Yamaoka, S., Nosaka, T., Maki, M., and Hatanaka, M. (1990). Oncogenic transformation by the tax gene of human T-cell leukemia virus type I in vitro. Proc. Natl. Acad. Sci. U.S.A. 87, 1071-1075. doi: 10.1073/pnas.87.3.1071

The T- and B-cell Malignancy Study Group. (1988). The third nation-wide study on adult $\mathrm{T}$ cell leukemia/lymphoma (ATL) in Japan: characteristic patterns of HLA antigen and HTLV-I infection in ATL patients and their relatives. Int. J. Cancer 41, 505-512. doi: 10.1002/ijc.2910410406

Uchiyama, T., Yodoi, J., Sagawa, K., Takatsuki, K., and Uchino, H. (1977). Adult T-cell leukemia: clinical and hematologic features of 16 cases. Blood 50, 481-492.

Yamaguchi, K., Takatsuki, K., Seiki, M., and Yoshida, M. (1984). Adult T-cell leukemia virus (ATLV or HTLV) proviral DNA for the classification of T-cell malignancies. Rinsho Ketsueki 25, 547-553.

Yamaoka, S., Courtois, G., Bessia, C., Whiteside, S. T., Weil, R., Agou, F., et al. (1998). Complementation cloning of NEMO, a component of the IkappaB kinase complex essential for NF-kappaB activation. Cell 93 , 1231-1240. doi: 10.1016/S0092-8674 (00)81466-X

Yamaoka, S., Inoue, H., Sakurai, M., Sugiyama, T., Hazama, M., Yamada, T., et al. (1996). Constitutive activation of NF-kappa B is essential for transformation of rat fibroblasts by the human T-cell leukemia virus type I Tax protein. EMBO J. 15, 873-887.

Zhi, H., Yang, L., Kuo, Y. L., Ho, Y. K., Shih, H. M., and Giam, C. Z. (2011). NF-кB hyperactivation by HTLV-I tax induces cellular senescence, but can be alleviated by the viral anti-sense protein HBZ. PLoS Pathog. 7:e10 02025. doi: 10.1371/journal.ppat.10 02025

Conflict of Interest Statement: The authors declare that the research was conducted in the absence of any commercial or financial relationships that could be construed as a potential conflict of interest.

Received: 30 July 2013; accepted: 05 September 2013; published online: 23 September 2013.

Citation: Ren $T$ and Cheng $H$ (2013) Differential transforming activity of the retroviral Tax oncoproteins in human $T$ lymphocytes. Front. Microbiol. 4:287. doi: 10.3389/fmicb.2013.00287

This article was submitted to Virology, a section of the journal Frontiers in Microbiology.

Copyright (C) 2013 Ren and Cheng. This is an open-access article distributed under the terms of the Creative Commons Attribution License (CC BY). The use, distribution or reproduction in other forums is permitted, provided the original author(s) or licensor are credited and that the original publication in this journal is cited, in accordance with accepted academic practice. No use, distribution or reproduction is permitted which does not comply with these terms. 